“C 2019 IEEE. Personal use of this material is permitted. Permission from IEEE must be obtained for all other uses, in any current or future media, including reprinting/republishing this material for advertising or promotional purposes, creating new collective works, for resale or redistribution to servers or lists, or reuse of any copyrighted component of this work in other works." 


\title{
Effective Capacity Analysis of Multiuser Ultra-Dense Networks with Cell DTx
}

\author{
Qing $\mathrm{Li}^{*}$, Yu Chen*, Qimei Cui*, Yu Gu* and Guoqiang $\mathrm{Mao}^{\dagger}$ \\ *National Engineering Laboratory for Mobile Network Technologies, Beijing University of Posts and Telecommunications \\ Email: \{yu.chen, cuiqimei, liqing1994\}@bupt.edu.cn \\ ${ }^{\dagger}$ School of Computing and Communications, University of Technology Sydney \\ Email: g.mao@ieee.org
}

\begin{abstract}
Cell discontinuous transmission (Cell DTx) improves network performance because it help mitigate inter-cell interference. However, the relationship between this technology and network performance has been little studied. The aim of this work is to understand the impact of Cell DTx on users' quality of service $(\mathrm{QoS})$ performance of multiuser ultra-dense networks (UDNs). We extend the traditional one-dimensional effective capacity model and develop a new multidimensional framework for UDNs with Cell DTx, which can be applied to different scheduling policies. Under the round-robin and the max-C/I scheduling examples, computer simulation shows that the analytical and simulation results are in good agreement and thus validate the accuracy of our proposed framework.
\end{abstract}

\section{INTRODUCTION}

The cell densification is a key feature in $5 \mathrm{G}$ ultra-dense networks (UDNs) to enhance network throughput [1]. When base stations (BSs) are densely deployed and operating in the same frequency spectrum [2], severe inter-cell interference (ICI) is caused and a common technique to reduce ICI is the use of the cell discontinuous transmission (Cell DTx) technology. Moreover, 5G systems allow multiple users to share a channel and are required to support quality-of-service guarantees for different services. Therefore, it is important to understand the stochastic behavior of QoS performance of multiuser UDNs with Cell DTx.

A number of work has studied Cell DTx in improving the physical-layer spectral efficiency [3], [4] and energy efficiency performance [5], [6]. Polignano et al. [7] studied the impact of Cell DTx on the link-layer QoS performance of Voice over IP traffic under dynamic and semi persistent packet scheduling strategies by simulations. In order to mathematically analyze the QoS performance, such as queue length distribution and delay-violation probability, some work adopts a cross-layer effective capacity model [8]-[10]. Under full-buffer scenarios, Weyres et al. [11] analyzed the effective capacity in multiuser networks under the round-robin (RR) and the max-C/I scheduling policies. Cui et al. [12] calculated the maximum sustainable data rate of a link in UDNs under QoS constraints. $\mathrm{Gu}$ et al. [13] considered the burstiness of traffic in UDNs and derived the effective capacity in single-user scenarios. However, Cell DTx and multiuser scenarios are not jointly considered in those work.
The aim of this work is to quantitatively analyze the effective capacity and QoS performance with Cell DTx in multiuser UDNs. In particular, we continue Gu's work and develop a new analysis framework to approximate QoS performance of UDNs with Cell DTx, which could be applied to multiuser scenarios under the RR and the max-C/I scheduling policies.

The rest of this paper is organized as follows: Section II describes the multiuser UDNs model. Preliminaries on the effective capacity model is given in Section III. In Section IV, we develop a multidimensional effective capacity framework to analyze the QoS performance of UDNs with Cell DTx. In Section V, we apply the proposed framework to derive the approximation of the effective capacity of multiuser scenarios under the RR and the max-C/I scheduling policies. In Section VI, we build a simulation platform to validate the accuracy of our proposed framework. Section VII summarizes our work.

\section{SySTEM Model}

\section{A. System Architecture}

A system model example of a multiuser UDN is shown in Fig. 1. The network consists of several SBSs, each serves multiple user equipments (UEs) in a shared channel. The interference experienced by a UE is assumed to be caused by the most adjacent SBS [11], so the network can be simplified to a two-SBS model. The set of SBSs is denoted by $\mathcal{N}=\{1,2\}$; the set of UEs in SBS $n(n \in \mathcal{N})$ is denoted by $\mathcal{J}_{n}=\left\{1,2, \cdots, J_{n}\right\}$, where $J_{n}$ is the total number of associated UEs.

Each UE is assigned an infinite-sized buffer in its associated SBS. SBSs apply a specific scheduling policy to allocate the shared time resource among multiple UEs.

All the SBSs use Cell DTx, i.e., each SBS has two modes, namely an idle mode and an active mode [14]. If the buffer of the scheduled UE is empty and there is no data arrival at this slot, then the SBS switches to the idle mode; otherwise, it stays in the active mode for the target UE's data transmission. Fig. 2 illustrates the transition between these modes.

\section{B. Inter-Cell Interference Modeling}

We assume that the wireless channels experience block Rayleigh fading and channel conditions of UEs in SBS 


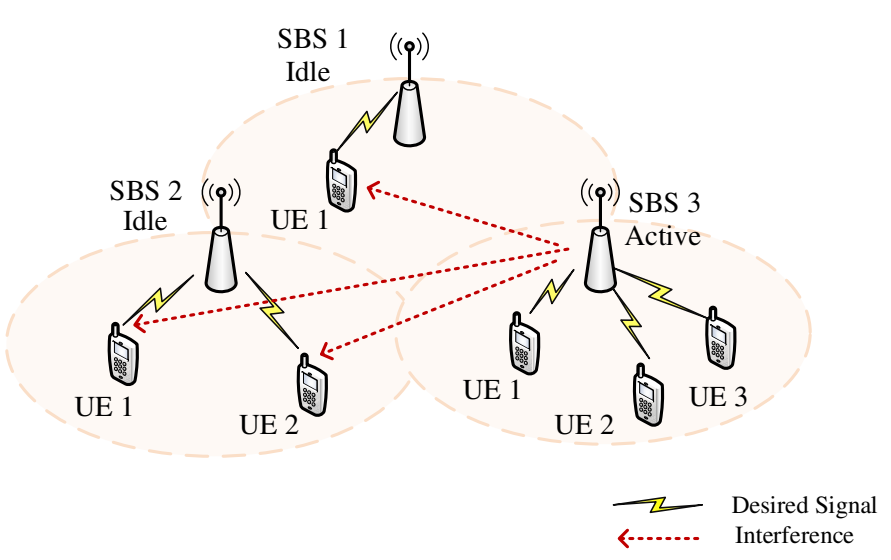

Fig. 1: An example of the system model consisting three SBSs and corresponding UEs. SBS 3 is active to transmit the data while SBS 1 and SBS 2 are in the idle mode.

$n$ are mutually independent. Let's consider UEs' signal-tointerference-plus-noise ratio (SINR) distribution in SBS $n$ $(n \in \mathcal{N})$. Let $\mathcal{M}$ denote the set of active SBSs at a given slot. The average received power of UEs in SBS $n$ is assumed to be equal and is denoted by $P_{n}^{S}$; the average interference power received by UEs in SBS $n$ from another active SBS $i(i \in \mathcal{M} \backslash n)$ is assumed to be equal and is denoted by $P_{i}^{I}$. Therefore, UEs in SBS $n$ have the same SINR distribution. The received SINR of UE $j$ in $\operatorname{SBS} n$ can be written as

$$
\gamma_{n, j}=\frac{P_{n}^{S}\left|H_{n, j}\right|^{2}}{\sum_{i \in \mathcal{M} \backslash n} P_{i}^{I}\left|H_{i, j}\right|^{2}+N_{0} B}\left(j \in \mathcal{J}_{n}\right),
$$

where $N_{0}$ is the noise spectral density, $B$ is the channel bandwidth, $H_{i, j}$ is the Rayleigh fading coefficient and $\left|H_{i, j}\right|^{2}$ is the channel gain exponentially distributed with unit mean. $\sigma^{2}=N_{0} B$ is the power of the additive white Gaussian noise.

The service $S_{n, j}[k](k=1,2,3 \cdots)$ is the amount of bits that SBS $n$ can transmit to UE $j$ at slot $k$. The service sequence $S_{n, j}[1], S_{n, j}[2] \cdots$ are independent and identically distributed (i.i.d.) random variables (RVs) identical to a RV $S_{n, j}$. According to Shannon's theorem, $S_{n, j}$ is a function of the bandwidth $B$, the duration of a slot $T_{s}$ and the SINR $\gamma_{n, j}$ :

$$
S_{n, j}=B T_{s} \log _{2}\left(1+\gamma_{n, j}\right) .
$$

Because UEs in SBS $n$ have the same SINR distribution and (2) holds, their service $S_{n, 1}, S_{n, 2}, \cdots, S_{n, J_{n}}$ follow an identical distribution.

\section{Traffic Model}

We make the following assumptions for UEs' traffic characters in SBS $n(n \in \mathcal{N})$ [15]:

1) The arrivals of UE $j\left(j \in \mathcal{J}_{n}\right)$ in $\operatorname{SBS} n$, $A_{n, j}[1], A_{n, j}[2], \ldots$ are i.i.d. RVs identical to a RV $A_{n, j}$;

2) The arrivals of UEs in SBS $n$ confirm to a same Bernoulli process with a data arrival probability $p_{n}$, $\left(p_{n} \in(0,1]\right)$;

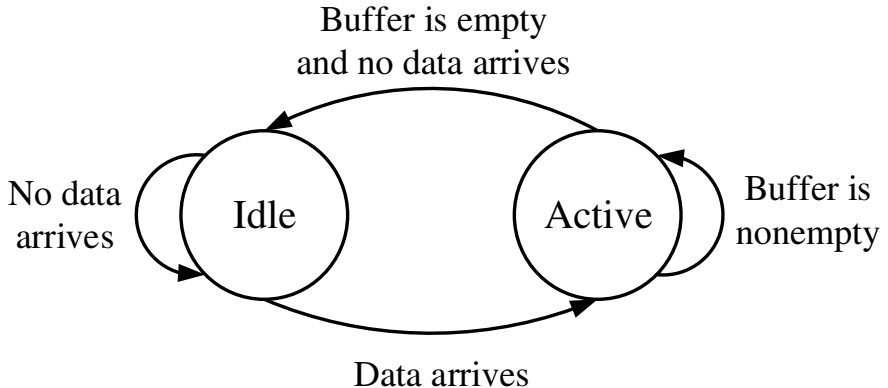

Fig. 2: Transition between two modes of a SBS with Cell DTx.

3) The arrival data lengths of UEs in SBS $n$ follow the same exponential distribution with a mean value $L_{n}$.

Based on the above assumptions, the arrivals of UEs in SBS $n, A_{n, 1}, A_{n, 2}, \cdots, A_{n, J_{n}}$ are i.i.d. RVs identical to a RV $A_{n}$. The probability distribution function (PDF) of $A_{n}$ is

$$
f_{A_{n}}(a)=\left\{\begin{array}{cc}
p_{n} \frac{1}{L_{n}} e^{-\frac{1}{L_{n}} a} & (a>0) \\
1-p_{n} & (a=0)
\end{array} .\right.
$$

$\mu_{n, j}$ is the average arrival rate of UE $j$ in SBS $n$. Based on (3), $\mu_{n, 1}, \mu_{n, 2}, \cdots, \mu_{n, J_{n}}$ equal a single value $\mu_{n}$ :

$$
\mu_{n, j}=\mu_{n}=L_{n} p_{n} / T_{s}\left(j \in \mathcal{J}_{n}\right) .
$$

\section{Preliminaries: One-Dimensional EfFective CAPACITY MODEL}

In the context of the traditional one-dimensional effective capacity model, the arrival and service rates of UE $j$ in SBS $n(n \in \mathcal{N})$ can be characterized by their effective bandwidth $\alpha_{n, j}^{(b)}\left(u_{n, j}\right)$ and effective capacity $\alpha_{n, j}^{(c)}\left(u_{n, j}\right)$ [15]:

$$
\begin{aligned}
\alpha_{n, j}^{(b)}\left(u_{n, j}\right) & =\frac{\Lambda_{A_{n, j}}\left(u_{n, j}\right)}{u_{n, j} T_{s}} \\
& =\frac{1}{u_{n, j} T_{s}} \log \left(\frac{p_{n}}{1-u_{n, j} L_{n}}+1-p_{n}\right), \\
\alpha_{n, j}^{(c)}\left(u_{n, j}\right) & =-\frac{\Lambda_{S_{n, j}}\left(-u_{n, j}\right)}{u_{n, j} T_{s}},\left(j \in \mathcal{J}_{n}\right)
\end{aligned}
$$

where $u_{n, j}$ is the QoS exponent, $\Lambda_{A_{n, j}}\left(u_{n, j}\right)$ and $\Lambda_{S_{n, j}}\left(-u_{n, j}\right)$ are the log-moment generating functions of $A_{n, j}$ and $S_{n, j}$, respectively. Let $C_{n, j}$ denote the service rate of UE $j$ in SBS $n$. By adopting the work by Soret et. al. [16], (6) can be approximated by a simplified form:

$$
\alpha_{n, j}^{(c)}\left(u_{n, j}\right) \approx \mathrm{E}\left[C_{n, j}\right]-\frac{\operatorname{Var}\left(C_{n, j}\right) T_{s}}{2} u_{n, j}
$$

where $\mathrm{E}[\cdot]$ is an expectation operator and $\operatorname{Var}(\cdot)$ is a variance operator. If there is a unique QoS exponent $u_{n, j}^{*}>0$ that satisfies

$$
\alpha_{n, j}^{(b)}\left(u_{n, j}^{*}\right)=\alpha_{n, j}^{(c)}\left(u_{n, j}^{*}\right)
$$


then the complementary cumulative distribution function (CCDF) of the quene length $Q_{n, j}$ can be approximated by [16]

$$
P\left(Q_{n, j}>B_{n, j}\right) \approx p_{b}^{n, j} e^{-u_{n, j}^{*} B_{n, j}},
$$

where $P(\cdot)$ is the probability of an event, $B_{n, j}$ is a queue length bound, $p_{b}^{n, j}$ is the nonempty buffer probability of the queue. According to the work [17]:

$$
p_{b}^{n, j} \approx 1-u_{n, j}^{*} L_{n}
$$

Denote $D_{n, j}$ as the steady-state delay experienced by a flow of UE $j$ in SBS $n$. According to the work [15], $D_{n, j}$ is a RV and its CCDF can be approximated as

$$
\begin{aligned}
P\left(D_{n, j}>t_{n, j}\right) & \approx p_{b}^{n, j} e^{-u_{n, j}^{*} \alpha_{n, j}^{(b)}\left(u_{n, j}^{*}\right) t_{n, j}} \\
& \approx\left(\frac{1-u_{n, j}^{*} L_{n}}{1-u_{n, j}^{*} L_{n}+p_{n} u_{n, j}^{*} L_{n}}\right)^{\frac{t_{n, j}}{T_{s}}+1}
\end{aligned}
$$

where $t_{n, j}$ is a delay bound.

\section{Multidimensional EFFECtive CAPACity FRAMEWORK FOR UDNS WITH CELL DTX}

In order to analyze the impact of Cell DTx on QoS performance, we extend the traditional one-dimensional effective capacity model and develop a new multidimensional framework for UDNs with Cell DTx. We first studied the SBS idle probability in IV-A, then derive the multidimensional effective capacity and analyze the QoS performance in IV-B.

\section{A. Analysis of the SBS Idle Probability}

As discussed in Section II-B and II-C, the arrival and service of UEs in SBS $n(n \in \mathcal{N})$ are both identically distributed, it can be implied from (5) - (8) that the unique QoS exponents of UEs in SBS $n, u_{n, 1}^{*}, u_{n, 2}^{*}, \cdots, u_{n, J_{n}}^{*}$ are equal to $u_{n}^{*}$ :

$$
u_{n, j}^{*}=u_{n}^{*}\left(j \in \mathcal{J}_{n}\right)
$$

Based on (10) and (12), the nonempty buffer probability of the queues in $\operatorname{SBS} n, p_{b}^{n, 1}, p_{b}^{n, 2}, \cdots, p_{b}^{n, J_{n}}$ are equal to $p_{b}^{n}$ :

$$
p_{b}^{n, j}=p_{b}^{n}\left(j \in \mathcal{J}_{n}\right)
$$

The idle probability of SBS $n$ is denoted by $P_{n}$. It is equivalent to 1) the probability that the scheduled queue is empty meanwhile 2) no data arrives at the queue [15]. As these two events are mutually independent, $P_{n}$ can be written as a product of the empty buffer probability $1-p_{b}^{n}$ and nonarrival probability $1-p_{n}$ :

$$
P_{n}=\left(1-p_{b}^{n}\right)\left(1-p_{n}\right) \approx u_{n}^{*} L_{n}\left(1-p_{n}\right) .
$$

\section{B. Multidimensional Effective Capacity Approximation}

As discussed in Section II-A, the network has two SBSs: SBS 1 and SBS 2. We firstly choose SBS 1 as the target SBS. According to discussion made in Section II-B and IV-A, the SINR distribution of UE $j\left(j \in \mathcal{J}_{1}\right)$ in SBS 1 is a function of the idle probability of SBS 2, which is $P_{2}$. From (2), (7) and (13), we find that the effective capacity of UE $j$ in SBS 1 is a function of both QoS exponents: $u_{1}$ and $u_{2}$. Applying the similar steps, we could also draw the conclusion that the effective capacity of $\mathrm{UE} r\left(r \in \mathcal{J}_{2}\right)$ in SBS 2 is also a function of both $u_{1}$ and $u_{2}$. Therefore, the key of analyzing the QoS performance is to find the unique QoS exponents $u_{1}^{*}, u_{2}^{*}>0$ that satisfy the following equations:

$$
\left\{\begin{array}{l}
\alpha_{1, j}^{(c)}\left(\mathbf{u}^{*}\right)=\alpha_{1, j}^{(b)}\left(u_{1}^{*}\right) \\
\alpha_{2, r}^{(c)}\left(\mathbf{u}^{*}\right)=\alpha_{2, r}^{(b)}\left(u_{2}^{*}\right)
\end{array},\right.
$$

where $\mathbf{u}^{*}=\left[u_{1}^{*}, u_{2}^{*}\right]$ is a unique QoS exponent vector. Eq. (15) can be solved by numerical methods, such as Newton's method. Moreover, it is discussed in the work [17] that the unique QoS exponent $u_{n}^{*}$ should always be less than $1 / L_{n}$. Therefore, an algorithm combining Newton's method and the bisection method is developed and expressed in pseudocode as follows:

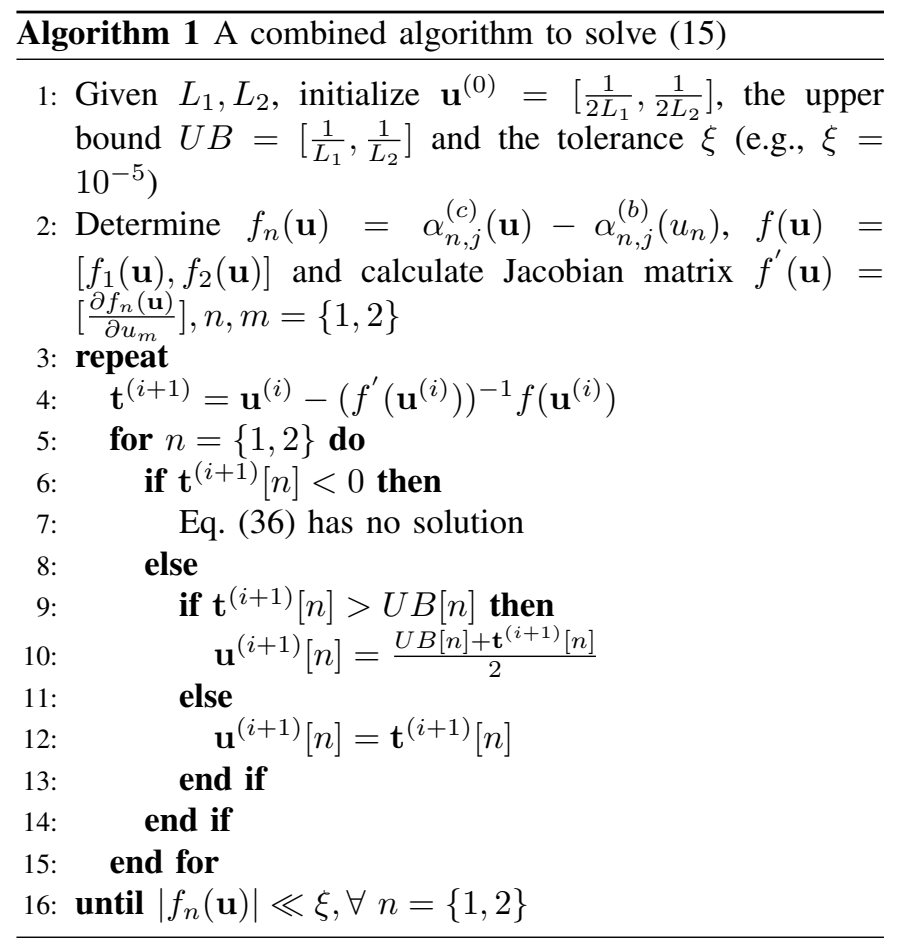

\section{EXAmples: EfFEctive CAPACITy OF Multiuser UDNS WITH CELL DTX}

In this section, we use the multidimensional effective capacity framework in Section IV-B to analyze the QoS performance of multiuser UDNs with Cell DTX. We first approximate the effective capacity of single-user scenarios in 
$\mathrm{V}-\mathrm{A}$ and then consider multiuser scenarios under the RR and the max-C/I scheduling policies in $\mathrm{V}-\mathrm{A}$ and $\mathrm{B}$, respectively.

\section{A. Effective Capacity of Single-User Scenarios}

We choose SBS 1 as the target SBS for the rest of the discussion. The SINR of the single UE in SBS 1 may have two cases:

Case I: SBS 2 is in a idle mode and causes no interference to the UE in SBS 1 with probability $P_{2} \approx u_{2}^{*} L_{2}\left(1-p_{2}\right)$. From (1), the UE's received SINR is

$$
\gamma_{1,1}^{(1)}=\frac{P_{1}^{S}\left|H_{1,1}\right|^{2}}{\sigma^{2}} .
$$

Because $\left|H_{1,1}\right|^{2}$ is exponentially distributed with unit mean, the PDF of the SINR is given by

$$
f_{\gamma_{1,1}^{(1)}}(\gamma)=\frac{\sigma^{2}}{P_{1}^{S}} e^{-\frac{\sigma^{2} \gamma}{P_{1}^{S}}}
$$

Case II: SBS 2 is in a active mode with probability $1-P_{2} \approx$ $1-u_{2}^{*} L_{2}\left(1-p_{2}\right)$. From (1), the UE's received SINR is

$$
\gamma_{1,1}^{(2)}=\frac{P_{1}^{S}\left|H_{1,1}\right|^{2}}{P_{2}^{I}\left|H_{2,1}\right|^{2}+\sigma^{2}} .
$$

Therefore, the PDF of the SINR is given by [13]

$$
f_{\gamma_{1,1}^{(2)}}(\gamma)=\left(\frac{\sigma^{2}}{P_{1}^{S}+P_{2}^{I} \gamma}+\frac{P_{1}^{S} P_{2}^{I}}{\left(P_{1}^{S}+P_{2}^{I} \gamma\right)^{2}}\right) e^{-\frac{\sigma^{2} \gamma}{P_{1}^{S}}} .
$$

By combing these two cases, the PDF of the UE's received SINR is a function of $u_{2}^{*}$ :

$$
\begin{aligned}
f_{\gamma_{1,1}}\left(\gamma, u_{2}\right) & =\left(P_{2} \frac{\sigma^{2}}{P_{1}^{S}}+\left(1-P_{2}\right)\right. \\
& \left.\cdot\left(\frac{\sigma^{2}}{P_{1}^{S}+P_{2}^{I} \gamma}+\frac{P_{1}^{S} P_{2}^{I}}{\left(P_{1}^{S}+P_{2}^{I} \gamma\right)^{2}}\right)\right) e^{-\frac{\sigma^{2} \gamma}{P_{1}^{S}}}
\end{aligned}
$$

The cumulative distribution function (CDF) of the SINR is an integral of (20):

$$
F_{\gamma_{1,1}}\left(\gamma, u_{2}\right)=1-\left(P_{2}+\left(1-P_{2}\right) \frac{P_{1}^{S}}{P_{1}^{S}+P_{2}^{I} \gamma}\right) e^{-\frac{\sigma^{2} \gamma}{P_{1}^{S}}}
$$

Denote $C_{1,1}\left(u_{2}\right)$ as the instantaneous service rate of the UE. The mean of $C_{1,1}\left(u_{2}^{*}\right)$ is the ergodic capacity:

$$
\begin{aligned}
\mathrm{E}\left[C_{1,1}\left(u_{2}\right)\right] & =\int_{0}^{+\infty} B \log _{2}(1+\gamma) f_{\gamma_{1,1}}\left(\gamma, u_{2}\right) d \gamma \\
& =\int_{0}^{+\infty} B \log _{2}(1+\gamma)\left(P_{2} \frac{\sigma^{2}}{P_{1}^{S}}+\left(1-P_{2}\right)\right. \\
& \left.\cdot\left(\frac{\sigma^{2}}{P_{1}^{S}+P_{2}^{I} \gamma}+\frac{P_{1}^{S} P_{2}^{I}}{\left(P_{1}^{S}+P_{2}^{I} \gamma\right)^{2}}\right)\right) e^{-\frac{\sigma^{2} \gamma}{P_{1}^{S}}} d \gamma
\end{aligned}
$$

The variance of $C_{1,1}\left(u_{2}\right)$ is

$$
\operatorname{Var}\left(C_{1,1}\left(u_{2}\right)\right)=\mathrm{E}\left[C_{1,1}^{2}\left(u_{2}\right)\right]-\mathrm{E}\left[C_{1,1}\left(u_{2}\right)\right]^{2} .
$$

\section{B. Effective Capacity Under the RR Scheduling}

Under the RR schduling, UEs are served in turns. Define the received SINR of UE $j$ in SBS 1 at slot $k(k=1,2,3 \cdots)$ under the RR scheduling $\gamma_{1, j ; \mathrm{RR}}[k]$ as follows:

$$
\gamma_{1, j ; \mathrm{RR}}[k]=\left\{\begin{array}{cc}
\gamma_{1, j}[k] & k \bmod J_{1}=j \\
0 & k \bmod J_{1} \neq j
\end{array} \quad\left(j \in \mathcal{J}_{1}\right) .\right.
$$

Denote $C_{1, j ; \mathrm{RR}}\left(u_{2}\right)$ as the service rate of UE $j$ in SBS 1 under the RR scheduling. Because SINR values are nonzero at certain slots (the $j^{\text {th }}$ slots for every $J_{1}$ slots), we make the following proposition:

Proposition 5.1: The mean and the variance of $C_{1, j ; \mathrm{RR}}\left(u_{2}\right)$ are

$$
\mathrm{E}\left[C_{1, j ; \mathrm{RR}}\left(u_{2}\right)\right]=\frac{1}{J_{1}} \mathrm{E}\left[C_{1,1}\left(u_{2}\right)\right]
$$

$\operatorname{Var}\left(C_{1, j ; \mathrm{RR}}\left(u_{2}\right)\right)=\frac{1}{J_{1}} \operatorname{Var}\left(C_{1,1}\left(u_{2}\right)\right)+\frac{J_{1}-1}{J_{1}^{2}} \mathrm{E}\left[C_{1,1}\left(u_{2}\right)\right]^{2}$.

For a proof of Proposition 5.1, see Appendix A. By substituting $\mathrm{E}\left[C_{n, j}\right]$ and $\operatorname{Var}\left(C_{n, j}\right)$ in (7) with (25) and (26), the effective capacity of UE $j$ in SBS 1 under the RR scheduling can be approximated by

$$
\begin{aligned}
& \alpha_{1, j ; \mathrm{RR}}^{(c)}\left(u_{1}, u_{2}\right) \approx \frac{1}{J_{1}} \mathrm{E}\left[C_{1,1}\left(u_{2}\right)\right] \\
& \quad-\frac{\left(\frac{1}{J_{1}} \operatorname{Var}\left(C_{1,1}\left(u_{2}\right)\right)+\frac{J_{1}-1}{J_{1}{ }^{2}} \mathrm{E}\left[C_{1,1}\left(u_{2}\right)\right]^{2}\right) T_{s}}{2} u_{1} .
\end{aligned}
$$

\section{Effective Capacity Under the Max-C/I Scheduling}

Under the max-C/I scheduling, UE with the instantaneously highest SINR is served [18]. The received SINR of UE $j$ in SBS 1 at slot $k(k=1,2,3 \cdots) \gamma_{1, j ; \max }[k]$ is defined as [19]:

$$
\gamma_{1, j ; \max }[k]=\left\{\begin{array}{cl}
\gamma_{1, j}[k] & \gamma_{1, j}[k]>\gamma_{1,-j}[k] \\
0 & \gamma_{1, j}[k]<\gamma_{1,-j}[k]
\end{array} \quad\left(j \in \mathcal{J}_{1}\right),\right.
$$

where $\gamma_{1,-j}[k]=\max _{m \in \mathcal{J}_{1}, m \neq j} \gamma_{1, m}[k]$ is the highest instantaneous SINR among UEs in SBS 1 except UE $j$. Because $\gamma_{1,-j}[k]$ are i.i.d. RVs identical to a $\mathrm{RV} \gamma_{1,-j}, \gamma_{1, j \text {;max }}[k]$ are i.i.d. RVs identical to a $\mathrm{RV} \gamma_{1, j ; \max }$. Denote $C_{1, j ; \max }\left(u_{2}\right)$ as the service rate of UE $j$ in SBS 1 under the max-C/I scheduling, which has the following properties:

Proposition 5.2: The mean and the variance of $C_{1, j ; \max }\left(u_{2}\right)$ are

$$
\begin{aligned}
& \mathrm{E}\left[C_{1, j ; \max }\left(u_{2}\right)\right]=\int_{0}^{+\infty} B \log _{2}(1+\gamma) \\
& \cdot\left(1-\left(\left(1-P_{2}\right) \frac{P_{1}^{S}}{P_{1}^{S}+P_{2}^{I} \gamma}+P_{2}\right) e^{-\frac{\sigma^{2} \gamma}{P_{1}^{S}}}\right)^{J_{1}-1} \\
& \cdot\left(P_{2} \frac{\sigma^{2}}{P_{1}^{S}}+\left(1-P_{2}\right)\left(\frac{\sigma^{2}}{P_{1}^{S}+P_{2}^{I} \gamma}+\frac{P_{1}^{S} P_{2}^{I}}{\left(P_{1}^{S}+P_{2}^{I} \gamma\right)^{2}}\right)\right) e^{-\frac{\sigma^{2} \gamma}{P_{1}^{S}}} d \gamma
\end{aligned}
$$

$\operatorname{Var}\left(C_{1, j ; \max }\left(u_{2}\right)\right)=\mathrm{E}\left[C_{1, j ; \max }^{2}\left(u_{2}\right)\right]-\mathrm{E}\left[C_{1, j ; \max }\left(u_{2}\right)\right]^{2}$. 


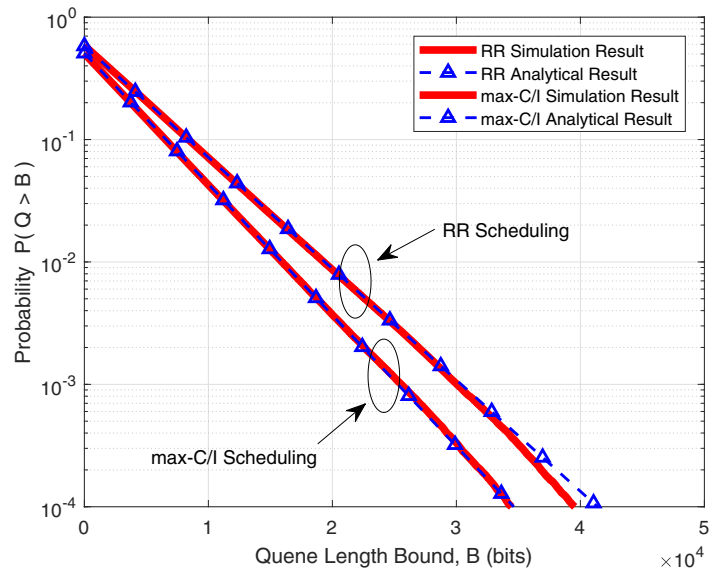

(a) Queue length distribution

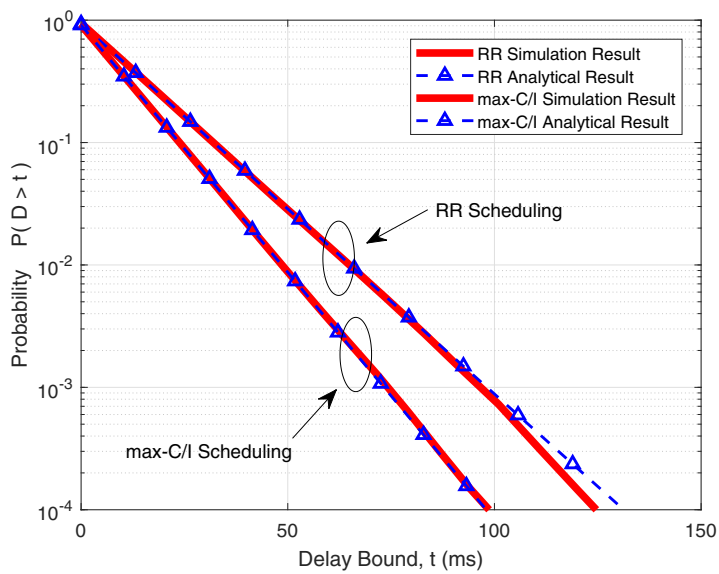

(b) Delay violation probability

Fig. 3: The queue length distribution $P(Q>B)$ and the delay-violation probability $P(D>t)$ of UE $j$ in SBS 1 under the $\mathrm{RR}$ and the max-C/I scheduling policies.

For a proof of Proposition 5.2, see Appendix B. By following the similar procedure in the analysis under the RR scheduling, the effective capacity of UE $j$ in SBS 1 under the max-C/I scheduling can be approximated as

$\alpha_{1, j ; \max }^{(c)}\left(u_{1}, u_{2}\right) \approx \mathrm{E}\left[C_{1, j ; \max }\left(u_{2}\right)\right]-\frac{\operatorname{Var}\left(C_{1, j ; \max }\left(u_{2}\right)\right) T_{s}}{2} u_{1}$.

By repeating the procedure from (24) to (31), the approximations of the effective capacity of UE $r$ in SBS $2\left(r \in \mathcal{J}_{2}\right)$ under the RR and the max-C/I scheduling are $\alpha_{2, r ; \mathrm{RR}}^{(c)}\left(u_{1}, u_{2}\right)$ and $\alpha_{2, r ; \max }^{(c)}\left(u_{1}, u_{2}\right)$, respectively.

Both Cell DTx and scheduling policies have no effect on the arrival process. Based on (5), the effective bandwidth of UEs in SBS 1 and SBS 2 are

$$
\begin{aligned}
& \alpha_{1, j ; \mathrm{RR} / \max }^{(b)}\left(u_{1}\right)=\frac{1}{u_{1} T_{s}} \log \left(\frac{p_{1}}{1-u_{1} L_{1}}+1-p_{1}\right) \\
& \alpha_{2, r ; \mathrm{RR} / \max }^{(b)}\left(u_{2}\right)=\frac{1}{u_{2} T_{s}} \log \left(\frac{p_{2}}{1-u_{2} L_{2}}+1-p_{2}\right) .
\end{aligned}
$$

We formulate the following nonlinear equations to find the unique QoS exponent vector $\mathbf{u}^{*}$ under the RR or the max-C/I scheduling policies:

$$
\left\{\begin{array}{l}
f_{1}(\mathbf{u})=\alpha_{1, j ; \mathrm{RR} / \max }^{(c)}(\mathbf{u})-\alpha_{1, j ; \mathrm{RR} / \max }^{(b)}\left(u_{1}\right) \\
f_{2}(\mathbf{u})=\alpha_{2, r ; \mathrm{RR} / \max }^{(c)}(\mathbf{u})-\alpha_{2, r ; \mathrm{RR} / \max }^{(b)}\left(u_{2}\right)
\end{array},\right.
$$

which could be solved by the algorithm summarized in Section IV-B.

\section{Results AND Discussions}

A simulation platform is built based on a two-SBS system model described in Section II-A. In our settings, SBS 1 serves three UEs while SBS 2 serves two UEs. The bandwidth $B$ is $180 \mathrm{kHz}$ that equals an LTE resource block; the duration of

\begin{tabular}{|c|c|}
\hline Parameter & Value \\
\hline Bandwidth, $B$ & $180 \mathrm{kHz}$ \\
\hline Noise spectral density, $N_{0}$ & $-174 \mathrm{dBm} / \mathrm{Hz}$ \\
\hline Duration of a slot, $T_{s}$ & $1 \mathrm{~ms}$ \\
\hline Number of associated UEs, $J_{n}$ & $J_{1}=3 ; J_{2}=2$ \\
\hline $\begin{array}{r}\text { Mean value of the arrival data } \\
\text { length, } L_{n}\end{array}$ & $L_{1}=2000 ; L_{2}=1000$ \\
\hline Data arrival probability, $p_{n}$ & $p_{1}=p_{2}=0.1$ \\
\hline $\begin{array}{r}\text { Average received signal } \\
\text { power, } P_{n}^{S} \\
\end{array}$ & $P_{1}^{S}=P_{2}^{S}=-101.44 \mathrm{dBm}$ \\
\hline Average interference power, $P_{i}^{I}$ & $\begin{array}{l}P_{1}^{I}=-120.44 \mathrm{dBm} ; P_{2}^{I}= \\
-119.44 \mathrm{dBm}\end{array}$ \\
\hline Simulation time & 10000 seconds \\
\hline
\end{tabular}
a slot $T_{s}$ is $1 \mathrm{~ms}$, which is a transmission time interval in
TABLE I: Simulation Parameters

LTE networks. The noise spectral density $N_{0}$ is set to -174 $\mathrm{dBm} / \mathrm{Hz}$. The average arrival rate of UEs in two SBSs are 200 Kbps (medium-quality video call) and $100 \mathrm{Kbps}$ (typical voice call), respectively. The average value of UEs' SINR without the interference from the other SBS is $20 \mathrm{~dB}$. Table I lists all the simulation parameters used in our work.

Fig. 3(a) and Fig. 3(b) show simulation and analytical results of the queue length distribution $P(Q>B)$ and the delay-violation probability $P(D>t)$ of UEs in SBS 1 under the RR and the max-C/I scheduling policies, respectively. The unique QoS exponent vector $\mathbf{u}^{*}$ is numerically found by Algorithm 1 and the analytical results are calculated by (9) and (11). As illustrated in Fig. 3, the simulation and analytical results overlap with each other under both scheduling policies, indicating that our proposed framework can accurately approximate the effective capacity and QoS performance of multiuser UDNs with Cell DTx.

The effective capacity region of UEs in SBS 1 under both the RR and the max-C/I scheduling policies is shown in Fig. 


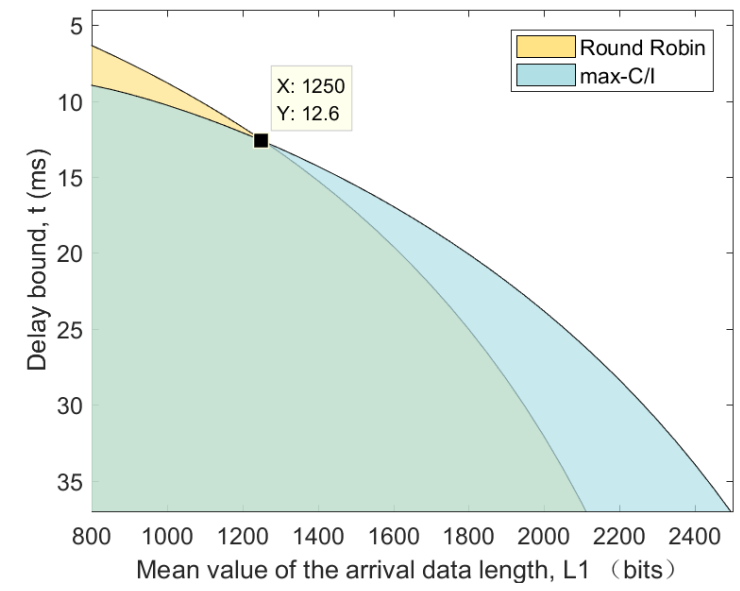

Fig. 4: The effective capacity region of UE $j$ in SBS 1 with $P(D>t)=0.1$ under the RR and the max-C/I scheduling policies

4. The $\mathrm{X}$-axis is the mean value of the arrival data length $L_{1}$ and the Y-axis is the corresponding delay bound $t$ with the delay violation constraint $P(D>t)=0.1$. With the same value of $L_{1}$, a smaller delay bound $t$ represent better QoS performance. From Fig. 4, we find that when $L_{1}>1250$ bits (high traffic load), the QoS performance under the max-C/I scheduling is much better than that of the RR. The reason is that when the traffic load is high, the QoS exponent $u_{1}^{*}$ approaches to zero. Therefore, from (7), the effective capacity is dominated by the mean of the service rate. Because a larger multiuser diversity is gained under the max-C/I scheduling, the service rate is much higher and the QoS performance is much better. However, when $L_{1}<1250$ bits (low traffic load), the QoS exponent $u_{1}^{*}$ approaches to $1 / L_{1}$ and the variance of the service rate dominates the effective capacity. Since the service rate at a certain time slot shows a higher randomness, the variance of the service rate is much higher and QoS performance is worse under the max-C/I scheduling than that of the RR. This regions shed important insights to understand the trade-off between the capacity and QoS performance of multiuser UDNs with Cell DTx.

\section{CONCLUSION}

Cell DTx is a key technology to mitigates inter-cell interference. This study has taken a step in understanding the QoS performance improvement with Cell DTx. In this paper, we propose a multidimensional effective capacity framework and develop to approximate QoS performance of UDNs with Cell DTx. We further give an example of using the framework to analyze multiuser scenarios under the round-robin and the max-C/I scheduling policies. Under both scheduling policies, the simulation and analytical results overlap with each other, validating the accuracy of our proposed framework. Our work reveals complex interactions among traffic, scheduling policy and QoS performance, which could be used to optimize network operations such as power control and resource allocation.

\section{ACKNOWLEDGMENT}

This paper is supported by the National Science and Technology Major Project No. 2018ZX030110004, the National Natural Science Foundation of China under Grant 61471058 and 61428102, and 111 Project of China B16006.

\section{APPENDIX A}

PROOF OF PROPOSITION 5.1

According to (2) and (24), only the service rates at the $j^{\text {th }}$ slots for every $J_{1}$ slots are nonzero. Therefore, the mean of $C_{1, j ; \mathrm{RR}}$ is

$$
\begin{aligned}
\mathrm{E}\left[C_{1, j ; \mathrm{RR}}\left(u_{2}\right)\right] & =\lim _{N \rightarrow \infty} \frac{1}{N} \sum_{k=1}^{N} C_{1, j ; \mathrm{RR}}\left(u_{2}\right)[k] \\
& =\frac{1}{J_{1}} \mathrm{E}\left[C_{1,1}\left(u_{2}\right)\right] .
\end{aligned}
$$

According to the definition, the variance of $C_{1, j ; \mathrm{RR}}\left(u_{2}\right)$ is

$$
\begin{aligned}
& \operatorname{Var}\left(C_{1, j ; \mathrm{RR}}\left(u_{2}\right)\right) \\
& =\lim _{N \rightarrow \infty} \frac{1}{N} \sum_{k=1}^{N}\left(C_{1, j ; \mathrm{RR}}\left(u_{2}\right)[k]-\mathrm{E}\left[C_{1, j ; \mathrm{RR}}\left(u_{2}\right)\right]\right)^{2} \\
& =\lim _{N \rightarrow \infty} \frac{1}{N}\left(\begin{array}{l}
\frac{\left(J_{1}-1\right) N}{J_{1}} \mathrm{E}\left[C_{1, j ; \mathrm{RR}}\left(u_{2}\right)\right]^{2} \\
+\sum\left(C_{1, j ; \mathrm{RR}}\left(u_{2}\right)[x]-\mathrm{E}\left[C_{1, j ; \mathrm{RR}}\left(u_{2}\right)\right]\right)^{2}
\end{array}\right),
\end{aligned}
$$

where

$$
x=j+m J_{1}, m=0,1,2, \cdots
$$

Denote $C_{1, j ; \mathrm{RR}}\left(u_{2}\right)[x]-\mathrm{E}\left[C_{1,1}\left(u_{2}\right)\right]=\Delta C_{1, j ; \mathrm{RR}}\left(u_{2}\right)[x]$. Because

$$
\lim _{N \rightarrow \infty} \sum_{m=0}^{N / J_{1}} \Delta C_{1, j ; \mathrm{RR}}\left(u_{2}\right)[x]=0,
$$

$$
\lim _{N \rightarrow \infty} \frac{1}{N} \sum_{m=0}^{N / J_{1}}\left(\Delta C_{1, j ; \mathrm{RR}}\left(u_{2}\right)[x]\right)^{2}=\frac{1}{J_{1}} \operatorname{Var}\left(C_{1,1}\left(u_{2}\right)\right)^{2}
$$

then the variance of $C_{1, j ; \mathrm{RR}}\left(u_{2}\right)$ is

$$
\begin{aligned}
& \operatorname{Var}\left(C_{1, j ; \mathrm{RR}}\left(u_{2}\right)\right)=\frac{J_{1}-1}{J_{1}}\left(\frac{\mathrm{E}\left[C_{1,1}\left(u_{2}\right)\right]}{J_{1}}\right)^{2} \\
& +\lim _{N \rightarrow \infty} \frac{1}{N} \sum_{m=0}^{N / J_{1}}\left(\Delta C_{1, j ; \mathrm{RR}}\left(u_{2}\right)[x]+\frac{J_{1}-1}{J_{1}} \mathrm{E}\left[C_{1,1}\left(u_{2}\right)\right]\right)^{2} \\
& =\frac{J_{1}-1}{J_{1}{ }^{3}} \mathrm{E}\left[C_{1,1}\left(u_{2}\right)\right]^{2}+\frac{1}{J_{1}} \operatorname{Var}\left(C_{1,1}\left(u_{2}\right)\right) \\
& +\frac{\left(J_{1}-1\right)^{2}}{J_{1}{ }^{2}} \mathrm{E}\left[C_{1,1}\left(u_{2}\right)\right]^{2} \\
& =\frac{1}{J_{1}} \operatorname{Var}\left(C_{1,1}\left(u_{2}\right)\right)+\frac{J_{1}-1}{J_{1}{ }^{2}} \mathrm{E}\left[C_{1,1}\left(u_{2}\right)\right]^{2} .
\end{aligned}
$$




\section{APPENDIX B}

PROOF OF PROPOSITION 5.2

The PDF of $\gamma_{1, j ; \max }$ can be found in the work [19]:

$$
\begin{aligned}
f_{\gamma_{1, j ; \max }}\left(\gamma, u_{2}\right)=P & \left(\gamma_{1, j}<\gamma_{1,-j}\right) \delta(\gamma) \\
& +F_{\gamma_{1,-j}}\left(\gamma, u_{2}\right) f_{\gamma_{1, j}}\left(\gamma, u_{2}\right),
\end{aligned}
$$

where $\delta(\gamma)$ is the Dirac delta function, $f_{\gamma_{1, j}}$ is the PDF of SINR of UE $j$ in SBS 1 in single-user scenarios. $F_{\gamma_{1,-j}}$ is the CDF of $\gamma_{1,-j}$. Based on the assumption that the channel conditions of UEs in the same SBS are mutually independent, $F_{\gamma_{1,-j}}$ can be derived according to order statistic [20]:

$$
\begin{aligned}
& F_{\gamma_{1,-j}}\left(\gamma, u_{2}\right)=\prod_{m \in \mathcal{J}_{1}, m \neq j} F_{\gamma_{1, m}}\left(\gamma, u_{2}\right) \\
& =\left(1-\left(\left(1-P_{2}\right) \frac{P_{1}^{S}}{P_{1}^{S}+P_{2}^{I} \gamma}+P_{2}\right) e^{-\frac{\sigma^{2} \gamma}{P_{1}^{S}}}\right)^{J_{1}-1} .
\end{aligned}
$$

By substituting $f_{\gamma_{1, j}}\left(\gamma, u_{2}\right)$ and $F_{\gamma_{1,-j}}\left(\gamma, u_{2}\right)$ in (41) with (20) and (42), the PDF of $\gamma_{1, j \text {;max }}$ can be written as

$$
\begin{aligned}
& f_{\gamma_{1, j ; \max }}\left(\gamma, u_{2}\right)=P\left(\gamma_{1, j}<\gamma_{1,-j}\right) \delta(\gamma) \\
& +\left(1-\left(\left(1-P_{2}\right) \frac{P_{1}^{S}}{P_{1}^{S}+P_{2}^{I} \gamma}+P_{2}\right) e^{-\frac{\sigma^{2} \gamma}{P_{1}^{S}}}\right)^{J_{1}-1} \\
& \cdot\left(P_{2} \frac{\sigma^{2}}{P_{1}^{S}}+\left(1-P_{2}\right)\left(\frac{\sigma^{2}}{P_{1}^{S}+P_{2}^{I} \gamma}+\frac{P_{1}^{S} P_{2}^{I}}{\left(P_{1}^{S}+P_{2}^{I} \gamma\right)^{2}}\right)\right) e^{-\frac{\sigma^{2} \gamma}{P_{1}^{S}}} .
\end{aligned}
$$

The mean of $C_{1, j ; \max }\left(u_{2}\right)$ is

$$
\begin{aligned}
& \mathrm{E}\left[C_{1, j ; \max }\left(u_{2}\right)\right] \stackrel{(1)}{=} \int_{0}^{+\infty} B \log _{2}(1+\gamma) f_{\gamma_{1, j} ; \max }\left(\gamma, u_{2}\right) d \gamma \\
& \stackrel{(2)}{=} \int_{0}^{+\infty} B \log _{2}(1+\gamma) P\left(\gamma_{1, j}<\gamma_{1,-j}\right) \delta(\gamma) d \gamma \\
& +\int_{0}^{+\infty} B \log _{2}(1+\gamma) F_{\gamma_{1,-j}}\left(\gamma, u_{2}\right) f_{\gamma_{1, j}}\left(\gamma, u_{2}\right) d \gamma \\
& \stackrel{(3)}{=} \int_{0}^{+\infty} B \log _{2}(1+\gamma) F_{\gamma_{1,-j}}\left(\gamma, u_{2}\right) f_{\gamma_{1, j}}\left(\gamma, u_{2}\right) d \gamma \\
& \stackrel{(4)}{=} \int_{0}^{+\infty} B \log _{2}(1+\gamma) \\
& \cdot\left(1-\left(\left(1-P_{2}\right) \frac{P_{1}^{S}}{P_{1}^{S}+P_{2}^{I} \gamma}+P_{2}\right) e^{-\frac{\sigma^{2} \gamma}{P_{1}^{S}}}\right)^{J_{1}-1} \\
& \cdot\left(P_{2} \frac{\sigma^{2}}{P_{1}^{S}}+\left(1-P_{2}\right)\left(\frac{\sigma^{2}}{P_{1}^{S}+P_{2}^{I} \gamma}+\frac{P_{1}^{S} P_{2}^{I}}{\left(P_{1}^{S}+P_{2}^{I} \gamma\right)^{2}}\right)\right) e^{-\frac{\sigma^{2} \gamma}{P_{1}^{S}}} d \gamma .
\end{aligned}
$$

The $3^{\text {rd }}$ equality in (44) follows due to the integral property of the Dirac delta function, i.e., the result of $\int_{0}^{+\infty} B \log _{2}(1+$ $\gamma) P\left(\gamma_{1, j}<\gamma_{1,-j}\right) \delta(\gamma) d \gamma$ equals zero by substituting $\gamma=$ 0 into $B \log _{2}(1+\gamma) P\left(\gamma_{1, j}<\gamma_{1,-j}\right)$. The variance of $C_{1, j ; \max }\left(u_{2}\right)$ is

$$
\operatorname{Var}\left(C_{1, j ; \max }\left(u_{2}\right)\right)=\mathrm{E}\left[C_{1, j ; \max ^{2}}\left(u_{2}\right)\right]-\mathrm{E}\left[C_{1, j ; \max }\left(u_{2}\right)\right]^{2} .
$$

\section{REFERENCES}

[1] X. Ge, S. Tu, G. Mao, C. X. Wang, and T. Han,"5G Ultra-Dense Cellular Networks,"IEEE Wirel. Commun., vol. 23, no. 1, pp. 7279, 2016.

[2] C. Yang, J. Li, Q. Ni, A. Anpalagan, and M. Guizani, "InterferenceAware Energy Efficiency Maximization in 5G Ultra-Dense Networks,“ IEEE Trans. Commun., vol. 65, no. 2, pp. 728-739, Feb.2017.

[3] R. Bonnefoi, C. Moy, and J. Palicot, "New macrocell downlink energy consumption minimization with cell DTx and power control,"IEEE Int. Conf. Commun., 2017.

[4] P. Chang and G. Miao, "Energy and Spectral Efficiency of Cellular Networks with Discontinuous Transmission," IEEE Trans. Wirel. Commun., vol. 16, no. 5, pp. 2991-3002, 2017.

[5] G. Andersson, A. Vastberg, A. Devlic, and C. Cavdar, "Energy efficient heterogeneous network deployment with cell DTx," in 2016 IEEE International Conference on Communications (ICC), pp.1-6, 2016.

[6] H. Holtkamp, G. Auer, S. Bazzi, and H. Haas, "Minimizing base station power consumption" IEEE J. Sel. Areas Commun., vol. 32, no. 2, pp. 297306, 2014.

[7] M. Polignano, D. Vinella, D. Laselva, J. Wigard, and T. B. Srensens, "Power savings and QoS impact for VoIP application with DRX / DTX feature in LTE, "IEEE Veh. Technol. Conf., 2011.

[8] D. Wu and R. Negi, "Effective capacity: a wireless link model for support of quality of service," IEEE Trans. Wirel. Commun., vol. 2, no. 4, pp. 630-643, 2003.

[9] Q. Cui, Y. Gu, W. Ni, and R. Liu, "Effective Capacity of LicensedAssisted Access in Unlicensed Spectrum for 5G: From Theory to Application“, IEEE J. Sel. Areas Commun., vol. 35, no.8, pp. 17541767, 2017.

[10] T. Abrao, S. Yang, L. D. H. Sampaio, P. J. E. Jeszensky, and L. Hanzo,"Achieving maximum effective capacity in OFDMA networks operating under statistical delay guarantee" emphIEEE Access, vol. 5, pp. 1433314346, 2017.

[11] M. Weyres and J. Gross, "Effective Service Capacity Analysis of Interference-Limited Multi-Carrier Wireless Systems,“ Wirel. Conf. (EW), Proc. 2013 19th Eur., pp. 1-7, 2013.

[12] Q. Cui, Y. Gu, W. Ni, X. Zhang, X. Tao, P. Zhang, R. Liu, "Preserving Reliability of Heterogeneous Ultra-Dense Distributed Networks in Unlicensed Spectrum,“ IEEE Commun. Mag.,vol. 56, no. 6, pp. 7278, 2018.

[13] Y. Gu, Q. Cui, Y. Chen, W. Ni, X. Tao and P. Zhang, "Effective Capacity Analysis in Ultra-Dense Wireless Networks with Random Interference,“ IEEE ACCESS, vol. 6, pp. 19499-19508, 2018.

[14] R. Bonnefoi, C. Moy, H. Farès, and J. Palicot, "Power Allocation for Minimizing Energy Consumption of OFDMA Downlink with Cell DTx," 2017 24th International Conference on Telecommunications $(I C T)$, no. $1,2017$.

[15] J. Xu, Y. Chen, Q. Cui, and X. Tao, "Use of Two-Mode Transceiver Circuitry and Its Cross-Layer Energy Efficiency Analysis,“ IEEE Commun. Lett., vol. 21, no. 9, pp. 2065-2068, 2017.

[16] B. Soret, M. Aguayo-Torres, and J. Entrambasaguas, "Capacity with explicit delay guarantees for generic sources over correlated rayleigh channel,“ IEEE Trans. Wirel. Commun., vol. 9, no. 6, pp. 1901-1911, 2010.

[17] Y. Chen and I. Darwazeh, "An Accurate Approximation of Delay with Nakagami-m Channels and Exponential Arrivals," 2015 IEEE Global Communications Conference (GLOBECOM), pp. 1-6, 2015.

[18] E. Dahlman and J. Sko, 4G LTE/LTE-Advance for Mobile Broadband, Second Edition, 2014.

[19] J. Perez, J. Ibanez, and I. Santamaria, "Exact closed-form expressions for the sum capacity and individual users' rates in broadcast ergodic rayleigh fading channels, “ 2007 IEEE 8th Workshop on Signal Processing Advances in Wireless Communications, pp. 1-5, 2007.

[20] A. Papoulis and S.U. Pillai, Probability, random variables, and stochastic processes. McGraw-Hill electrical and electronic engineering series. McGraw-Hill, 2002. 